\title{
"EFFECT OF OCIMUM IN CHEMOTHERAPY INDUCED OXIDATIVE STRESS" A STUDY CONDUCTED IN DAKSHINA KANNADA DISTRICT, KARNATAKA, INDIA
}

Debkumar Ray, Sumana Chaudhury

1. Assistant Professor, Department of Biochemistry, B. S. Medical College, Bankura, West Bengal.

2. PG Student, Department of Botany, Burdwan Universiy, Burdwan, West Bengal.

\section{CORRESPONDING AUTHOR}

Dr. Debkumar Ray,

Assistant Professor, Dept of Biochemistry,

KL-1, Jagatpur, Ganga-Yamuna Apartment,

Block-B, Flat: B/C\&D,

Ashwininagar,Kolkata-700059

E-mail: dr.debkumar@gmail.com,

Phone: 00919476233354.

ABSTRACT: Adriamycin (ADR) belongs to anthracycline group of antibiotics, which is widely used for the complete remission of solid tumours. In carcinoma breast, non Hodgkin's lymphoma and Hodgkin's lymphoma, ADR is considered as most useful chemotherapeutic medication. Anthracycline by virtue of their quinone group generates free radicals in solution in both normal and malignant cells. The Ocimum leaf extract as well as their flavonoids orientin and vicenin have strong antioxidant activity in vitro and antilipid peroxidative effect in vivo, and strongly suggest free radical scavenging as a major mechanism by which Ocimum products protect against cellular damage and tumour induction. However, most interest has been devoted to the antioxidant activity of flavonoids, which is due to their ability to reduce free radical formation and to scavenge free radicals. The capacity of flavonoids to act as antioxidants in vitro has been a subject of several studies in the past years. The antioxidant efficacy of flavonoids present in Ocimum sanctum in vivo is less documented, presumably because of the limited knowledge on their uptake in tumours. Inspite of limitation of the knowledge of exact mechanism of uptake of flavonoids we have studied antioxidant efficacy of Ocimum sanctum aqueous extract $(140 \mathrm{mg}$ ) with placebo control in carcinoma breast, non Hodgkin's lymphoma, Hodgkin's lymphoma cases along with chemotherapy particularly during third cycle. Statistical analysis has been done by Mann Whitney's test which shows no significant difference of antioxidant enzymes between study $(n=19)$ and control $(n=16)$ group before chemotherapy. There is a significant increase of all scavenging enzymes in study group $(n=19)$ compared to control group $(n=16)$ after chemotherapy. Significant increase in the Haemoglobin content and enhanced activities of Superoxide dismutase ( SOD) and Catalase (CAT) in the study group which was given Ocimum.

KEY WORDS: Ocimum sanctum, Adriamycin, Antioxidants, Free radicals, Superoxide dismutase, Catalase

INTRODUCTION: In last 25 years, a large number of studies have shown that free radical mediated reactions are responsible for a wide range of chemotherapy induced side effects, and that antioxidants are able to protect non malignant cells against some of the damaging effect of cytotoxic drugs(1).Inspite of many available natural and synthetic antioxidants, a few number have been studied in clinical trials. Beta carotene, Vitamin C and E, azelastine, co-enzyme Q10,

Journal of Evolution of Medical and Dental Sciences/Volume1/Issue5/November-2012Page-864 
selenium have been studied for their chemotherapeutic effects and found to be effective in ameliorating the side effects of anticancer drugs. Natural products like silymarin(1), ginseng(2), ashwagandha(3) are found to be beneficial adjuncts to chemotherapy. There is a concern that potent antioxidants may interfere with the anticancer efficacy too, which however appears to be a theoretical concern since many studies done using both chemotherapy and antioxidants have shown just the opposite. Antioxidant supplements have shown to protect the normal cells while making the cancer cells more vulnerable to cytotoxicity of chemotherapy and radiation therapy(1).The unpretentious little backyard plant plays on essential role in the folk medicine of South Asia named "Tulsi" (Ocimum sanctum) in Sanskrit, is a gentle therapeutic herb belonging to the family Basil. Conventionally, it is supposed to guard against stress and modern research now corroborates the concept (4).In laboratory animals, Ocimum sanctum has been shown to prevent cancer and to protect against radiation damage. Study established that hamsters were protected from developing cancer of oral cavity by Ocimum leaf extract(5).Mice survived radiation exposure when they have given with the herb $(6,7,8)$.Its historical use as immunostimulant has now been demonstrated(4).Two flavonoids have been identified in Ocimum leaves, Orientin and Vicenin(6). In vivo and vitro studies have shown that, in general, flavonoids have antioxidant (9), antitumour promoter (10), antimetastatic (11) and anti-proliferative (12) properties. Flavonoids may act as antioxidants either by directly scavenging the free radicals or by modulating the free radical scavenging enzymes in the body(4).The direct free radical scavenging action of Ocimum aqueous extract(13) and indirectly by modulating Superoxide dismutase, Catalase and other antioxidant enzymes like Glutathione peroxidase(14).in its protective role in radiation induced damage in mice ,have been established .In human beings, Ocimum has been studied for its efficacy in non Insulin dependent Diabetes Mellitus (NIDDM)(15) but not for its chemotherapeutic effects. However, Ocimum aqueous extract is undergoing clinical trial currently for its radiation protective effect at Tata Memorial Hospital, Mumbai.

Our aim of study is to evaluate the safety and efficacy of Ocimum aqueous extract in modifying the side effects of Adriamycin (ADR) based chemoschedules in the treatment of post operative breast cancer, non Hodgkin's lymphoma and Hodgkin's lymphoma.

MATERIALS AND METHODS: This is a simple randomized single blind placebo control study, where the study group was having 19 and control group includes 16 cases of post operative female breast cancer, non Hodgkin's lymphoma, Hodgkins lymphoma receiving Adriamycin based chemoschedules and Statistical analysis has been done by Mann Whitney's test which shows no significant difference of antioxidant enzymes between study $(n=19)$ and control $(n=16)$ group before chemotherapy, but after chemotherapy it was significant . The study was undertaken in Department of Biochemistry in association with Department of Radiotherapy and Radiobiology in Kasturba Medical College, Mangalore, India.

INCLUSION CRITERIA: Post operative breast cancer in women, non Hodgkin's and Hodgkin's lymphoma in either gender, cancer diagnosed on both clinical and histological basis; age below 55 years, normal laboratory safety profile range, normal Electrocardiogram(ECG),general well being as assessed by Karnofsky's performance status scale more than 70;willing to give the written informed consent. 
EXCLUSION CRITERIA: Haemoglobin less than $10 \mathrm{~g} / \mathrm{dl}$, WBC less than 3,000/ml, Platelet count less than $80,000 / \mathrm{ml}$ grossly damaged Liver and renal function tests more than thrice of the normal range, congestive cardiac failure, hypertension, uncontrolled diabetes mellitus.

STUDY DRUGS: Placebo capsule contains lactose.

Ocimum capsule contains $140 \mathrm{mg}$ dried aqueous extract of Ocimum leaves. The fresh leaves of Ocimum shades dried and powdered. The aqueous extract was prepared by refluxing with distilled water and was vacuum dried in a speed vac system prepared in Radiobiology Department, KMC, Manipal. The dose has been selected on the basis of the animal experiments done in same department.

TREATMENT PLAN AND ASSESSMENT OF ENDPOINT MEASURES: Patients who are willing to give written informed consent were enrolled for the study, before which the clinical examination and laboratory assessment of organ function tests were done to rule out the exclusion criteria. Patients were randomized to either of the groups to receive the placebo or Ocimum capsules. The study medication was given during $3^{\text {rd }}$ cycle, 24 hours after chemotherapy for six consecutive days. Superoxide dismutase (SOD), Catalase (CAT) and Haemoglobin $(\mathrm{Hb})$ was estimated before chemotherapy and 24 hours after the end of Ocimum / Placebo adjuvant therapy of the same cycle on $7^{\text {th }}$ day. Estimation of Haemoglobin was done by cyanmethaemoglobin method $(16,17)$.Superoxide dismutase (SOD) was determined by Mccord and Fridovich method (18) and Catalase (CAT) activity was assessed by Brannan et al (19) method.

RESULTS: The baseline SOD values of the two groups are not significantly different. The SOD levels in the study group and control group were $6814.66 \pm 3540.04$ and $9321.83 \pm 5887.23$. However the control group had a higher mean value of SOD than the study group. The values of SOD levels and the change in SOD levels obtained after $3^{\text {rd }}$ cycle of chemotherapy are given in Table 3.

The mean value of SOD in the control group was $6422.94 \pm 3854.10 \mathrm{U} / \mathrm{g}$ Hb while study groups showed a value of $9892.46 \pm 4227.60 \mathrm{U} / \mathrm{g} \mathrm{Hb}$. There was a significant difference in the change in SOD after chemotherapy between the two groups. The mean change in SOD in the study group was 3077.79 while the control group showed a fall in SOD value with the mean change in the value of -2898.89 .

Figure 1 and 2 depicts the mean values of SOD obtained before and after $3^{\text {rd }}$ cycle of chemotherapy in the study and control groups.

The mean values of CAT levels estimated before $3^{\text {rd }}$ cycle of chemotherapy are presented in table 4

There is no significant difference in the baseline Catalase values of the two groups. However the study group showed an apparently higher mean value of Catalase in comparison with with control group (Figure 3).Values of CAT and the change in CAT observed after $3^{\text {rd }}$ cycle of chemotherapy are given in table 5

A significant increase in the Catalase levels was observed in the study group with the mean value of $49,727.72 \pm 18,264.80$. The control group showed a significant decrease and the mean value was $10,016.83 \pm 7547.10$.There was a significant difference in the change in Catalase values after chemotherapy between the two groups. Study group showed a difference of $28,826.69$ and the control group showed a mean value of -3477.19. Figure 3 and 4 depicts the

Journal of Evolution of Medical and Dental Sciences/Volume1/Issue5/November-2012Page-866 
mean values of CAT obtained before and after $3^{\text {rd }}$ cycle of chemotherapy in the study and control groups

Table 1 Karnofsky's Performance Status (KPS)

\begin{tabular}{|l|c|}
\hline KPS & Description of the general condition of the patients \\
\hline $100 \%$ & Normal,no complaints,no evidence of disease \\
\hline $90 \%$ & Able to carry on normal activity;minor signs or symptoms of disease \\
\hline $80 \%$ & Nornal activity with effort;some signs and symptoms of disease \\
\hline $70 \%$ & Cares for self;unable to carry on normal activityor to do active work \\
\hline $60 \%$ & Requires occational assistance,but is mostly able to carefor himself \\
\hline $50 \%$ & Requires considerable assistance and frequent medical care \\
\hline $40 \%$ & Disabled,requires special care and assistance \\
\hline $30 \%$ & Severely disabled,hospitalization indicated;death not imminent \\
\hline $20 \%$ & Very sick,hospitalization necessary active supportive treatment \\
\hline $10 \%$ & Moribund,fatal processes,progressing rapidly \\
\hline $0 \%$ & Dead \\
\hline
\end{tabular}

Table 2 : SOD levels before $3^{\text {rd }}$ cycle of chemotherapy

\begin{tabular}{|c|c|c|}
\hline & $\mathrm{n}$ & Mean \pm SD(Unit/g Hb) \\
\hline Study Group & 19 & $6814.66 \pm 3540.04$ \\
\hline Control Group & 16 & $9321.83 \pm 5887.23$ \\
\hline
\end{tabular}

Table 3 : SOD levels after $3^{\text {rd }}$ cycle of chemotherapy

\begin{tabular}{|c|c|l|l|}
\hline & $\mathrm{n}$ & Mean \pm SD $(\mathrm{U} / \mathrm{gHb})$ & Change in SOD \\
\hline Study Group & 19 & $9892.46 \pm 4227.60$ & $3077.79 \pm 2325.68^{* *}$ \\
\hline Control Group & 16 & $6422.94 \pm 3854.10$ & $-2898.89 \pm 3183.50$ \\
\hline
\end{tabular}

Table 4 : CAT levels before $3^{\text {rd }}$ cycle of chemotherapy

\begin{tabular}{|c|c|c|}
\hline & $\mathrm{n}$ & Mean \pm SD(U/g Hb) \\
\hline Study Group & 19 & $20.901 .03 \pm 9901.13$ \\
\hline Control Group & 16 & $13494.03 \pm 7476.94$ \\
\hline
\end{tabular}

Table 5 : CAT levels after $3^{\text {rd }}$ cycle of chemotherapy

\begin{tabular}{|l|l|l|l|}
\hline & $\mathrm{n}$ & Mean \pm SD $(\mathrm{U} / \mathrm{g} \mathrm{Hb})$ & Change in Catalase \\
\hline Study Group & 19 & $49,727.27 \pm 18264.80^{* *}$ & $28,826.69 \pm 25536.24^{* * *}$ \\
\hline Control Group & 16 & $10,016.83 \pm 7547.10$ & $-3477.19 \pm 2946.16$ \\
\hline
\end{tabular}

$\mathrm{n}=$ number of subjects

** p value 0.002

$* * * \mathrm{p}$ value 0.001 
Figure 1 : Comparison of SOD(Unit/gHb) levels between study and control group before $3^{\text {rd }}$ cycle of chemotherapy

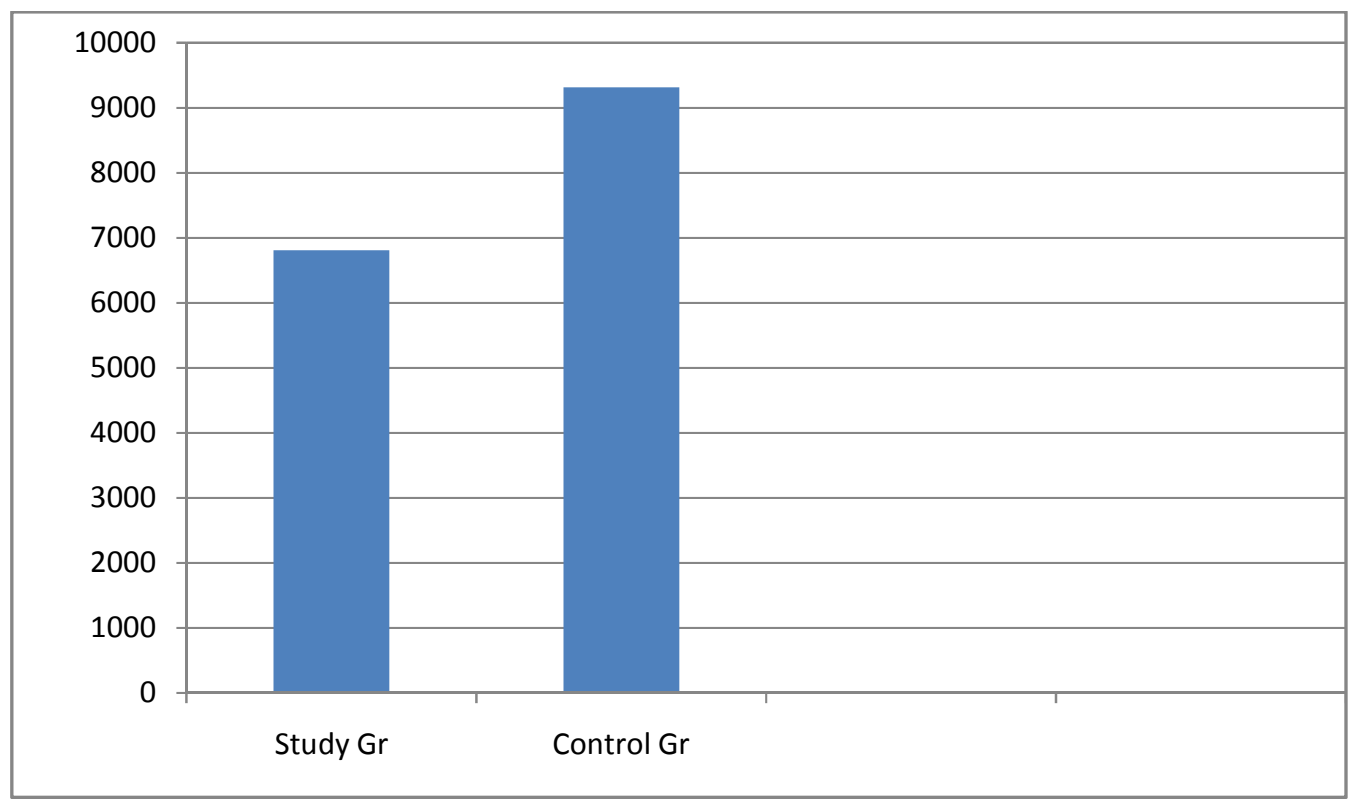

Figure 2 : Comparison of SOD(Unit/gHb) levels between study and control group after $3^{\text {rd }}$ cycle of chemotherapy

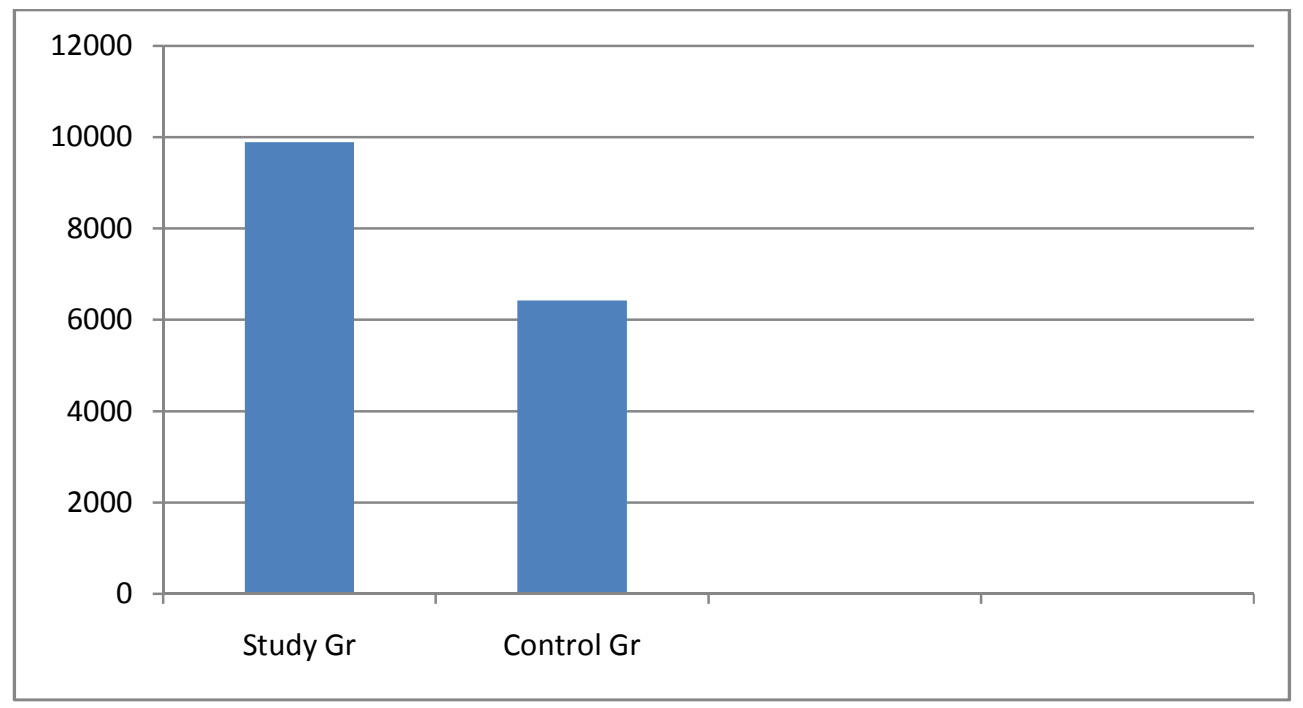

Figure 3 : Comparison of CAT(Unit/gHb) levels between study and control group before $3^{\text {rd }}$ cycle of chemotherapy 


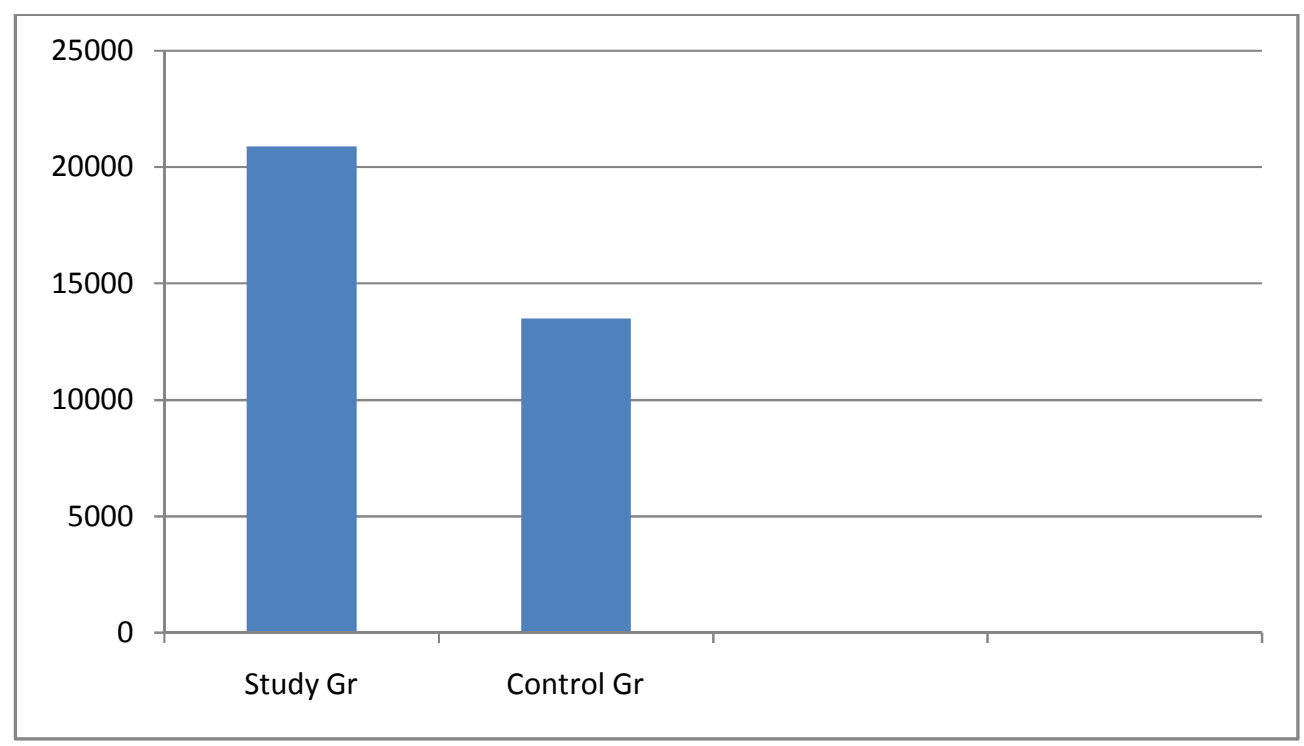

Figure 4 : Comparison of CAT(Unit/gHb) levels between study and control group after $3^{\text {rd }}$ cycle of chemotherapy

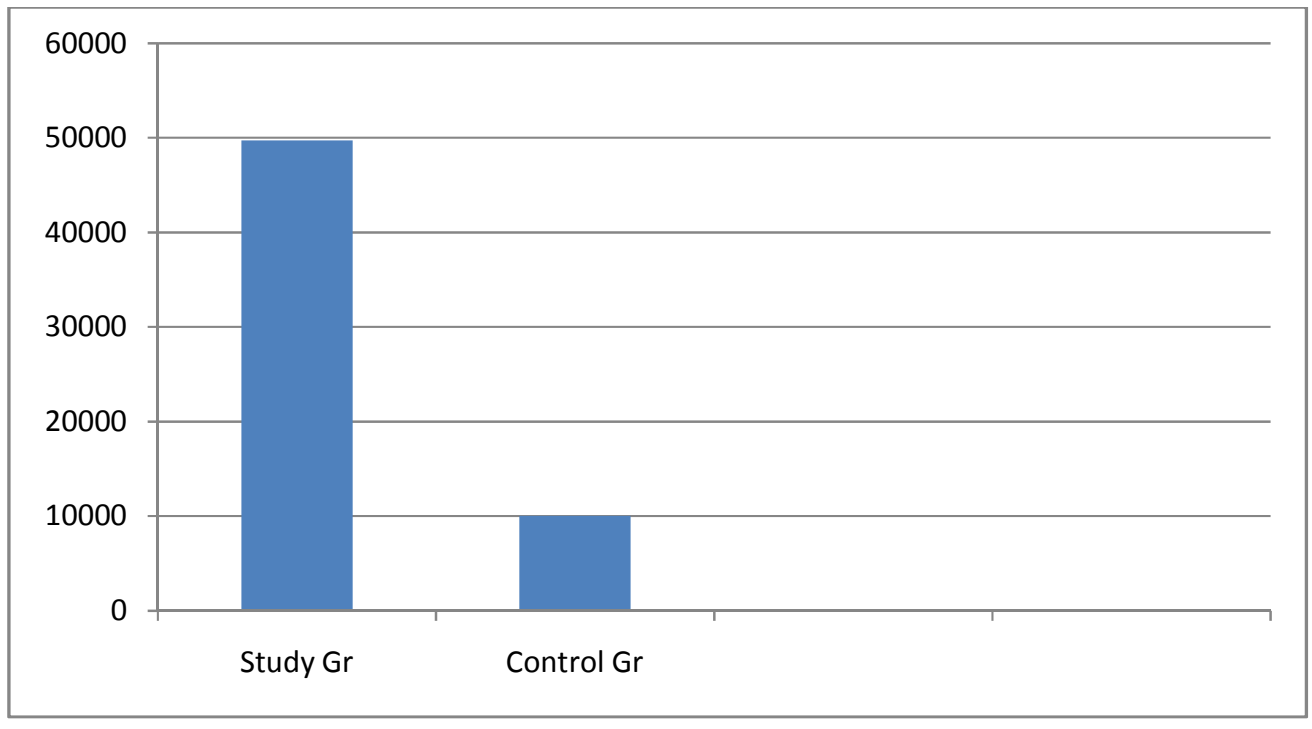

DISCUSSION: Chemical toxins produce biological damage by forming reactive oxygen species like singlet oxygen, superoxides, hydroxyl, hydroperoxy, hydrogen peroxides (20).Ikeda et al.(21) have shown that anthracycline by virtue of their quinone groups generate free radicals in solution in both normal and malignant cells. In addition generation of lipid peroxide nitric oxide and other destructive radicals are also produced from semiquinone intermediates of anthracyclines. Under normal conditions the inherent defence system including SOD,CAT and other antioxidant enzymes protect against free radical mediated oxidative stress.

$\mathrm{Cu}-\mathrm{Zn}$ SOD serves as a major antioxidant enzyme in erythrocytes where superoxide radicals are continuously generated by auto oxidation of Haemoglobin. Several anticancer drugs lead to a generation of reactive oxygen species which partly explains the cytotoxic effect of cancer therapy. 
Increase in SOD activity was $44 \%$ in the study group treated with Ocimum extract. However placebo treated group showed a $31 \%$ reduction in SOD activity suggesting an efficient scavenging of free radicals by Ocimum extract.

Uma devi et al (22) have shown that significant decrease in SOD activity after radiotherapy,but the activity increases following administration of Ocimum extract.

Our result is in agreement with the above study. Significant increase in SOD observed in Ocimum treated patients suggests that the Ocimum extract facilitates the removal of superoxide anions produced by chemotherapy.

Mammalian erythrocytes are endowed with extraordinarily high activities of Catalase. Cohen et al (23) have suggested that under physiological conditions practically all hydrogen peroxides encountered by erythrocytes are detoxified by glutathione peroxidase and that Catalase has a vital role in the clearance of hydrogen peroxide. Jacob et al (24) have reported that humans lacking erythrocyte Catalase activity are not even susceptible to hemolysis induced by oxidant drugs. Nihal et al (25) have reported protection by Catalase against exogenous oxidant challenge. A 2.3 fold increase in Catalase activity was observed in the study group treated with Ocimum extract where as placebo group showed 1.3 fold reduction in activity. A significant increase in Catalase activity observed ( $p$ value 0.001 ) suggests a brisk generation of superoxides and therefore hydrogen peroxide in the present circumstances. Flavinoids orientin and vicenin in Ocimum seems to have induced the activity of Catalase. Thus providing protection against the toxicity of anthracyclines.

Doroshow et al 1986(26) have shown that anthracycline induced cytotoxicity is ameliorated by adding SOD and CAT to the culture medium.

No significant change in Haemoglobin content observed in the two groups after chemotherapy. However the decrease in SOD, CAT observed in control group without any appreciable alteration in the Haemoglobin levels.

A significant increase in the SOD,CAT levels after chemotherapy along with Ocimum extract is an important observation of this study. This suggests that absorbed flavonoids of Ocimum may play an important role to promote the body enzymatic (SOD, CAT) defence system to combat the free radicals produced during ADR based chemotherapy. Thus Ocimum sanctum could be a beneficial adjuvant for the same.

The possibility of developing new chemoprotective for application from Ocimum sanctum should be further explored. In addition, its impressive antioxidant property and stimulatory effect on the cellular antioxidants can be exploited for adjuvant therapy against a number of human ailments such as cancer and other stress related disorders.

CONCLUSION: The results of present study indicate a significant elevation in the antioxidant enzymes viz.SOD and Catalase after chemotherapy along with Ocimum as an adjuvant.

Increased antioxidant activity brought about by Ocimum may be responsible to combat the damage in normal healthy tissues with little or no protection to the tumour following chemotherapy. Hence Ocimum can be a beneficial adjuvant with ADR based chemotherapy.

\section{REFERENCES:}

1. Weji NL,Cleton FL,Osanto S.Free radicals and antioxidants in chemotherapy induced toxicity.Cancer Treat Rev 1997;23:209-240

2. Torosian MH.Metabolic abnormalities and conditionally essential nutrients in the cancer patient. Adjuvant Nutrition in Cancer Treatment 1992;173-233. 
3. Ziauddin $\mathrm{N}$,et al.Studies on the immunomodulatoryeffects of ashwagandha.J of Ethnopharm, 1996;50(2):69-76

4. Tirtha,Sadashiva,Swami.The Ayurveda Encyclopedia 1998;106.

5. Kartkeyan K,Ravichandran P,Govindaswami S.Chemoprotective effectof ocimum sanctum on DMBA- Inducedhamster buccal pouch carcinogenesis.Oral Oncl 1999;35(1):112-119

6. Umadevi P,Ganasoundari A,Rao BS,Srinivasan KK.In vivo radioprotection by ocimum flavonoids:survival of mouse. Radiat res 1999;151(1):74-78

7. Umadevi P,Bisht KS,Vinitha MA. Comparative study of radioprotection by ocimum flavonoids and Synthetic aminothiol protectors in the mouse.Br.J Radiol 1998;71(847):782-784

8. Ganasoundari A,Mahamood Zare S,Uma Devi.Modification of bone marrow sensitivity by Medicinal Plant Extracts.The Br.J Radiol 1997;70:599-602

9. Alan L,Muller MD.Antioxidant Flavonoids:Structure,function and clinical usage.Alt Med Rev 1996;1(2):103-111

10. Fujiki $\mathrm{H}$ et al. Inhibition of tumour promotion by flavonoids.In plant flavonoids in biology and medicine. Biochemical,pharmacologicaland structure activity relationships Alan R.Liss.New York.1986;429-440

11. Bracke $\mathrm{ME}$ et al.Flavonoids inhibit malignant tumours invasion in vitro.In plant flavonoids in Biology and Medicine.II.Biochemical,Cellular and medicinal properties.Alan R.Liss.New York 1988;219-233

12. Kandaswamy $\mathrm{C}$ et al.Antiproliferative effects of citrus flavonoids on a human squamous cell carcinoma in vitro.Cancer Lett 1991;56:147-152

13. Ganasoundari A,Uma Devi P,Rao MNA.Protection against radiation induced chromosome damage in Mouse bone marrow by ocimum sanctum.Mutat Res 1997;373:271-276

14. Uma Devi P,Ganasoundari A.Modulation of glutathione and antioxidant enzymes by ocimum sanctum and its protection against radiation injury.Indian J Exp Biol 1999;Mar 37:262-268

15. Agarwal P,Rai V,Singh RB.Randomized,placebo controlled,single blind trial of holy ocimum leaves in Patientswith non insulin dependent diabetes mellitus.Int J Clin Pharmacol Ther 1996; Sept 34:406-409

16. Drabkin DL et al.J Biol Chem 1932;98:719

17. Zijlstra NC.Clinical Chem Acta 1960;5:719

18. McCord JM,Fridovich I.Superoxide dismutase :An enzymatic function for erythrocuprein.J.Biol Chem 1969;24:6049-6055

19. Brannan TS,Maker HO,Raes JP.Regional distribution of catalase in the adult rat brain.J Neurochem 1981; 86:307-309

20. Von Sonntag C.The chemical basis for radiation Biology (Taylor and Francis,London ). 1987

21. Ikeda K,Kajiwara K,Tanabi E,Tokumaru S.Kishida E,Masuzawa Y and Kojo S.Involvement of hydrogen peroxide and hydroxyl radical in chemically induced apoptosis of HL -60 cells.Biochem Pharmacol 1999;57:1361-1365

22. Younes Mand Siegers CP.Planta Medica 1981;43:240

23. Cohen,G and P.Hochstein.Glutathione peroxidase: the primary agent for the elimination of hydrogen peroxide in erythrocytes.Biochemistry:1963;2:1420-1428

24. Jacob,H.S.,S.H.Ingbar and J.H.Jandl.Oxidative hemolysis and erythrocyte metabolism in hereditary acatalasia.J.Clin.Invest.1965;44:1187-1199

25. Nihal S Agar,S.M.H.Sadrzadeh,Philip E.Hallaway and John W Eaton.Erythrocyte Catalase a somatic oxidant defence.J Clin Invest.1985;319-321

26. Doroshow JH,Role of hydrogen peroxide and hydroxyl radical formation in killing of Ehrlich tumour Cells by anticancer quinines,Proc Natl Acad Sci USA,1986;83:4514-4518 